\title{
Percepciones de alumnado y profesorado sobre una intervención de mobile learning en inglés como lengua extranjera
}

\section{Students' and teachers' perceptions of a mobile learning intervention in English as foreign language}

\author{
Yolanda Somoano García ${ }^{1}$, José Ignacio Menéndez Santurio ${ }^{2}$ \\ ${ }^{1}$ Colegio Montedeva, España (somoano.yolanda@gmail.com) \\ ${ }^{2}$ Universidad Isabel I, España (nachomenendeztibet@gmail.com)
}

Recibido el 21 de julio de 2017; revisado el 12 de septiembre de 2017; aceptado el 13 de noviembre de 2017; publicado el 1 de junio de 2018

\section{RESUMEN:}

En la actualidad, el empleo de las tecnologías móviles está ampliamente extendido. Debido a ello, en los últimos años ha habido un creciente interés por incluir estos elementos en la enseñanza $\mathrm{y}$ visibilizar sus posibilidades educativas. Sin embargo, aún son escasos los trabajos que han estudiado este aspecto en inglés como lengua extranjera en la etapa de educación secundaria. Por ello, el objetivo de este estudio fue el de conocer las percepciones de alumnado y profesorado sobre una intervención basada en mobile assisted language learning. Se utilizó un diseño de estudio de caso. 18 estudiantes de educación secundaria y una profesora participaron en la investigación. Se utilizaron diferentes instrumentos para la recogida de información: (1) diario del profesor, (2) pregunta abierta y (3) entrevistas semi-estructuras. Se empleó el programa Atlas.ti 7 para ayudar a la gestión de los datos. Todas las respuestas de los participantes fueron analizadas mediante métodos de comparación constante y análisis de contenido. El análisis de los datos produjo seis categorías: aprendizaje, novedad, cooperación, motivación, enfoque de enseñanza y amistad. Los resultados señalaron que la intervención ayudó al alumnado a mejorar sus competencias lingüísticas, su motivación por aprender inglés, a trabajar en equipo y a mejorar los lazos de amistad con sus compañeros. Asimismo, el planteamiento fue considerado novedoso, alejado del enfoque tradicional de enseñanza basado en el libro de texto. Como conclusión, estos resultados reflejan los beneficios educativos del aprendizaje mediante dispositivos móviles en la asignatura de inglés como lengua extranjera en educación secundaria.

\section{PALABRAS CLAVE: TIC, EDUCACIÓN SECUNDARIA, APRENDIZAJE MEDIANTE DISPOSITIVOS MÓVILES, INNOVACIÓN.}

\begin{abstract}
:
Nowadays, the use of mobile technologies is widely extended. As a consequence, over the last few years there has been an increasing interest in including these elements in teaching as well as in highlighting its educational possibilities. However, there is still little research that has studied this aspect in secondary education. Thus, the aim of this study was to know the students' and teachers' perceptions of a Mobile Assisted Language Learning intervention. A case study design was used. 18 secondary school students and a teacher participated in the research. Different instruments were used to collect data: (1) teacher's diary, (2) open-ended question and (c) semi-structured interviews. Atlas.ti 7 software was used to help with data management. All the participants' answers were analysed via content analysis and constant comparison methods. Data analysis produced six categories: learning, novelty, cooperation, motivation, teaching approach, and friendship. The results showed that the intervention helped students to improve their linguistic competence, their motivation for learning English, to work in teams, and to improve their friendship links
\end{abstract}


with their classmates. Likewise, the approach was considered as novel, far from the traditional approach based on the text book. In conclusion, these results reflect the educational benefits of mobile learning in english as a foreign language in secondary education.

KEYWORDS: ICT, SECONDARY SCHOOL, MOBILE LEARNING, INNOVATION.

\section{INTRODUCCIÓN}

Los avances tecnológicos tan importantes que se han producido en los últimos años han tenido un fuerte impacto en el devenir de la sociedad (Conole, 2016). En ese sentido, la educación y los procesos de enseñanza-aprendizaje que en ella se producen no han estado exentos de estas modificaciones (Illanas y Llorens, 2011). La irrupción de las tecnologías de la información y la comunicación (TIC) en este ámbito suponen un destacable punto de inflexión de carácter conceptual y metodológico que influye al modo en que las instituciones y el profesorado se enfrentan a los procesos educativos y a la gestión del aprendizaje (Amador y Pantoja, 2017; GarcíaPeñalvo y Seonae, 2015). Esto ha conllevado que en los últimos 40 años nos estemos enfrentando a una nueva ecología del aprendizaje que afecta a casi todos los elementos de este proceso: dónde, cuándo, con quién y de quién; cómo, qué y para qué se aprende (Coll, 2013).

La evolución tecnológica ha proporcionado un aparato que posee una gran potencialidad: el teléfono inteligente (smartphone), que junto con otros dispositivos como los MP3, los ordenadores portátiles o las tabletas, conforman los principales instrumentos del llamado aprendizaje móvil ( $m$ learning). El m-learning, según Shih y Mills (2007), podría definirse como:

La capacidad de aprendizaje en cualquier momento y en cualquier lugar a través del uso de la multimedia (texto, voz, imagen o vídeo) y la comunicación (llamada, mensajería de texto o voz, correo electrónico con acceso a la web). Este modo de enseñanza y aprendizaje proporciona la interacción en línea en tiempo real en una serie de actividades de aprendizaje cortas con características como las grabaciones de video o voz para contar historias o incluso para un diario de mobblogging. (p. 3)

Las características de los dispositivos del aprendizaje móvil son múltiples y variadas: portabilidad, interactividad, individualidad, conectividad, ubicuidad y adaptabilidad (Klopfer y Squire, 2008). Todas ellas lo convierten en tecnologías muy versátiles y con unas posibilidades educomunicativas muy trascendentales (Villalonga y Marta-Lazo, 2015).

La inclusión de estos dispositivos en el contexto educativo ha llevado a que muchos investigadores estudiaran sus potencialidades pedagógicas (Fombona y Pascual, 2013; Pastor y Zubillaga, 2012). En ese sentido, estos dispositivos se han revelado como herramientas que favorecen la creación de conocimiento, la mejora del trabajo en equipo, la posibilidad de acceder a información y contextos de aprendizaje a cualquier hora y momento del día, el fomento de las interacciones entre los diferentes agentes educativos o la promoción de un aprendizaje más activo y centrado en el alumnado, entre otros (Monguillot, González, Guitert y Zurita, 2014).

Uno de los enfoques derivados del aprendizaje móvil es el denominado mobile assisted language learning (MALL, aprendizaje de idiomas asistido por dispositivos móviles), entendido como la utilización de dispositivos móviles para el aprendizaje de un lenguaje (Lee y Lee, 2013). En ese sentido, el inglés como lengua extranjera (ILE; EGL, english as a foreign language) es una de las materias escolares en las que los dispositivos propios del aprendizaje móvil se han utilizado como herramientas para favorecer la adquisición del lenguaje (Liu, Yu, y Ran, 2008). A pesar de que, con anterioridad, una gran parte de los profesores de ILE han utilizado el computer assisted language learning (CALL, aprendizaje de idiomas asistido por computadores) (Kramsch, A'Ness, y Lam 2000), las limitaciones de este enfoque, especialmente en cuanto a portabilidad, y el creciente aumento del número de smartphones entre la población ha provocado un importante aumento en la utilización del MALL. Los resultados de la aplicación del MALL en ILE han reportado resultados destacables: mejoras en la comprensión y en la ortografía (Attewell, 2005), en la pronunciación (Arashnia y Shahrokhi, 2016), en la motivación hacia el aprendizaje del ILE (Lan, Sung, y Chang, 2007) y en la adquisición de vocabulario (Mulatu, 2015).

En el contexto de la asignatura de ILE, en la etapa educación secundaria, es habitual que los centros educativos prohíban la utilización de dispositivos móviles debido a "los malos usos que se supone que el alumnado hace del dispositivo" (Cantillo, Roura y Sánchez, 2012, p. 8). Esto hace que la escuela se aleje del mundo real del alumnado, los cuales viven inmersos en un mundo tecnológico fuera de las aulas, pero son obligados a abandonarlo una vez dentro de ellas (Cantillo et al., 2012). La 
prohibición de los dispositivos móviles en estos contextos es probablemente una de las causas por las que aún no está muy extendida la aplicación e investigación del MALL en ILE dentro de esta etapa. A pesar de que se tengan constancia de algunos estudios que han aplicado exitosamente intervenciones de MALL en ILE en educación secundaria (Hsu, Hwang y Chang, 2013; Lu, 2008), aún son necesarios más estudios que visibilicen todas sus potencialidades.

Por tanto, debido a los resultados positivos que se han obtenido de los escasos estudios que se han llevado a cabo, se hace necesario continuar analizando la aplicación de programas basados en MALL en educación secundaria dentro de la asignatura de ILE.

Teniendo en cuenta todo lo anterior, el objetivo de esta investigación fue conocer las percepciones de alumnado y profesorado sobre una intervención basada en MALL en la asignatura de ILE en la etapa de educación secundaria.

\section{MÉTODO}

\subsection{Participantes y diseño}

En la investigación participaron un total de 18 estudiantes (1 grupo natural de cuarto de la ESO), de entre 15 y 17 años de edad $(M=15,44$; $D T=, 61)$, de los cuales 10 eran mujeres y 8 hombres. También participó en el estudio la profesora encargada de llevar a cabo la intervención didáctica. Contaba con siete años de experiencia docente y no tenía experiencia previa en el uso de dispositivos móviles en ILE. Se utilizó un diseño de estudio de caso (Bisquerra, 2012).

\subsection{Instrumentos}

Diario del profesor. Con el objetivo de conocer los hechos más significativos de la experiencia, el profesor cumplimentó una entrada del diario al final de cada una de las sesiones. Cada entrada buscó dar respuesta a estas dos preguntas: pregunta 1 - ¿Qué ha sido lo más significativo que ha acontecido en la sesión de hoy? y pregunta 2 - ¿Por qué ha sido significativo?

Pregunta final abierta. Todos los estudiantes contestaron al final de la intervención la siguiente pregunta abierta: "Escribe lo que piensas, tus sensaciones y sentimientos sobre la UD de aprendizaje móvil".

Entrevistas semi-estructuradas. Al finalizar la intervención se realizaron diez entrevistas semiestructuradas a diferentes estudiantes. Los entrevistadores utilizaron un guion compuesto por diez preguntas que ayudaron a focalizar en los aspectos más significativos de estudio. Las entrevistas tuvieron una duración de entre 15 y 20 minutos.

\subsection{Programa de intervención}

La intervención didáctica se implementó en la asignatura de ILE a lo largo de 13 sesiones, de 55 minutos cada una. Para implementar el programa de intervención, el alumnado tuvo que traer a la clase sus propios dispositivos móviles para hacer uso de ellos a lo largo de toda la experiencia. Así pues, se optó por no utilizar el enfoque tradicional de enseñanza (libro de texto), sustituyéndolo por los dispositivos móviles y por tabletas que tenían acceso a Internet mediante el wifi del Centro. La utilización de los smartphones permitió ofrecer un enfoque de aprendizaje activo, centrado en el alumnado, y en el que el profesor actuó más como guía que como instructor. Así pues, las clases se caracterizaron por ser menos estructuradas y en las que los propios estudiantes gestionaban su tiempo de clase para la realización de las tareas. La mayoría de estas se llevaron a cabo en el aula, aunque en ocasiones se utilizaron otros espacios del Centro. En todo momento, la interacción entre el alumnado y la profesora fue en inglés.

Para el desarrollo de las actividades, los participantes se organizaron generalmente en parejas o en grupos reducidos de cuatro o cinco compañeros. Las actividades que se propusieron fueron interactivas, comunicativas y lingüísticamente útiles, con el objetivo de fomentar el trabajo en equipo, la organización y la adquisición de las diferentes destrezas de la lengua inglesa. Algunos de los elementos que se implementaron en esta intervención fueron: grabaciones de vídeo, entrevistas, podcast, etc. Cabe destacar que estos aspectos mencionados fueron seleccionados mediante Internet y siempre teniendo en consideración los niveles del alumnado de cada grupo/pareja. Con ello, se trató de dar cabida a un enfoque más autónomo y que tuviera en cuenta la diversidad del aula.

A lo largo de la experiencia, se diseñó un blog privado de la unidad didáctica, en la que solo el alumnado participante podía acceder. En este espacio el alumnado pudo compartir opiniones y sentimientos acerca de las actividades realizadas en clase, proponer recursos de interés sobre el inglés y, además, fue una importante forma de fomentar la interacción tanto entre los compañeros como con la profesora. Además de los diferentes aspectos citados 
con anterioridad, también se dieron uso diferentes aplicaciones que el alumnado tuvo que descargar en sus propios dispositivos móviles para llevar a cabo la experiencia. Algunas de estas fueron: un lector de códigos de Quick Response (QR), la aplicación Inigma, que sirve para leer códigos $\mathrm{QR}$ y la aplicación MyWordBook, creada por el British Council para fomentar el aprendizaje del vocabulario. En la tabla 1 se ofrece el plan actividades del programa de intervención.

Tabla 1. Plan de actividades

\begin{tabular}{|c|c|c|}
\hline Sesión & Materiales & Actividades \\
\hline 1 & $\begin{array}{l}\text { • Noticias que } \\
\text { contienen verbos } \\
\text { compuestos por } \\
\text { dos o más } \\
\text { palabras. } \\
\text { - Códigos QR. } \\
\text { • Inigma. } \\
\text { •MyWordBook. }\end{array}$ & $\begin{array}{l}\text { • Escanear códigos QR } \\
\text { con noticias que contienen } \\
\text { verbos compuestos por } \\
\text { dos o más palabras. } \\
\text { - Identificar los verbos } \\
\text { compuestos por dos o más } \\
\text { palabras, compartirlos con } \\
\text { los compañeros e } \\
\text { introducirlos en la } \\
\text { aplicación MyWordBook. } \\
\text { - Escribir un titular en } 140 \\
\text { caracteres o menos y } \\
\text { publicarlo en el blog. }\end{array}$ \\
\hline 2 & $\begin{array}{l}\text { - Actividades de } \\
\text { estilo indirecto en } \\
\text { línea. }\end{array}$ & $\begin{array}{l}\text { - Completar diferentes } \\
\text { actividades gramaticales } \\
\text { sobre estilo indirecto. }\end{array}$ \\
\hline $\begin{array}{c}3,4 \mathrm{y} \\
5\end{array}$ & - Podcasts. & $\begin{array}{l}\text { - Crear un repositorio de } \\
\text { podcasts: escuchar, } \\
\text { seleccionar, escribir } \\
\text { críticas y publicarlo en el } \\
\text { blog. }\end{array}$ \\
\hline 6 y 7 & $\begin{array}{l}\text { - Entrevistas con } \\
\text { personas famosas } \\
\text { en línea. }\end{array}$ & $\begin{array}{c}\text { - Escuchar e informar } \\
\text { sobre lo que dice la gente. }\end{array}$ \\
\hline $\begin{array}{l}8,9 \\
10 \\
11 \mathrm{y} \\
12\end{array}$ & $\begin{array}{c}\text { • Periódicos } \\
\text { nacionales, } \\
\text { internacionales, } \\
\text { deportivos y la } \\
\text { previsión del } \\
\text { tiempo en línea. }\end{array}$ & - Grabar un telediario. \\
\hline 13 & $\begin{array}{l}\text { - Códigos QR. } \\
\text { - Diferentes retos. }\end{array}$ & $\begin{array}{l}\text { - Caza del tesoro: } \\
\text { conseguir varios } \\
\text { elementos y completar } \\
\text { diferentes tareas. }\end{array}$ \\
\hline
\end{tabular}

\subsection{Procedimiento}

Para llevar a cabo la investigación, se obtuvo en primer lugar el permiso del equipo directivo del Centro. Posteriormente, se contactó con las familias de los participantes para informar a los padres y madres, así como para pedir su consentimiento. Tras ello, se llevó a cabo el programa de intervención a lo largo de 13 sesiones. Durante la intervención, la profesora-investigadora cumplimentó el diario del profesor y, al finalizar la unidad didáctica, todo el alumnado cumplimentó la pregunta abierta y diez alumnos fueron entrevistados de forma aleatoria. Se animó a los participantes a que contestaran lo más verazmente posible, informándoles de que sus datos serían tratados de forma confidencial y de que sus respuestas no influirían en la nota de la asignatura.

\subsection{Análisis de datos}

Respecto a los análisis cualitativos, se utilizó programa Atlas.ti 7 para la gestión de los datos. Las respuestas de los participantes fueron analizadas utilizando los métodos de comparaciones constantes (Libarkin y Kurdziel, 2002) y análisis de contenido (Denzin y Lincoln, 1994) para extraer diferentes categorías y patrones comunes en relación con cada caso y entre casos (Saldaña, 2009). Primeramente, los comentarios se leyeron en repetidas ocasiones para comprender el significado de la experiencia de manera general. La investigación cualitativa fue revisada por un experto en la materia para ayudar a mantener la objetividad del proceso durante el análisis de datos. Se identificaron diferentes categorías a medida que emergían naturalmente de las respuestas. Aquellas categorías resultantes del primer análisis independiente fueron examinadas críticamente por el experto por medio de un diálogo reflexivo que incluyó una discusión de las interpretaciones de las transcripciones. La fiabilidad del proceso se llevó a cabo por medio de un feedback constante y un análisis participativo por parte del investigador y el experto, revisando y depurando las categorías resultantes para que los resultados fueran considerados fiables y creíbles (Lincoln y Guba, 1985). El objetivo era hacer uso de la información recabada para aumentar la posible contribución de esta investigación a una comunidad educativa más amplia.

\section{RESULTADOS}

En este apartado, se muestran los resultados obtenidos de los datos cualitativos de la investigación (entrevistas y preguntas abiertas al alumnado, y el diario del profesor). Los datos se agrupan en torno a seis categorías principales que emergieron del análisis realizado: aprendizaje, novedad, cooperación, motivación, enfoque de enseñanza y amistad (figura 1). En la figura 2, se muestra la frecuencia de los extractos significativos de cada categoría. Posteriormente, se describen los resultados de cada una de las dimensiones 
resultantes de este estudio utilizando citas para ejemplificar.

\begin{tabular}{|c|c|c|} 
Entrevistas al alumnado & $\begin{array}{c}\text { NOVEDAD } \\
\text { Entrevistas al alumnado }\end{array}$ & $\begin{array}{c}\text { COOPERACIÓN } \\
\text { Entrevistas al alumnado } \\
\text { Pregunta abierta } \\
\text { Diario del profesor }\end{array}$ \\
\hline $\begin{array}{c}\text { Entrevistas al alumnado } \\
\text { Pregunta abierta } \\
\text { Diario del profesor }\end{array}$ & $\begin{array}{c}\text { PNegunta abierta } \\
\text { Entrevistas al alumnado } \\
\text { Pregunta abierta } \\
\text { Diario del profesor }\end{array}$ & $\begin{array}{c}\text { AMISTAD } \\
\text { Entrevistas al alumnado } \\
\text { Pregunta abierta } \\
\text { Diario del profesor }\end{array}$ \\
\hline
\end{tabular}

Figura 1. Categorías resultantes del análisis de datos

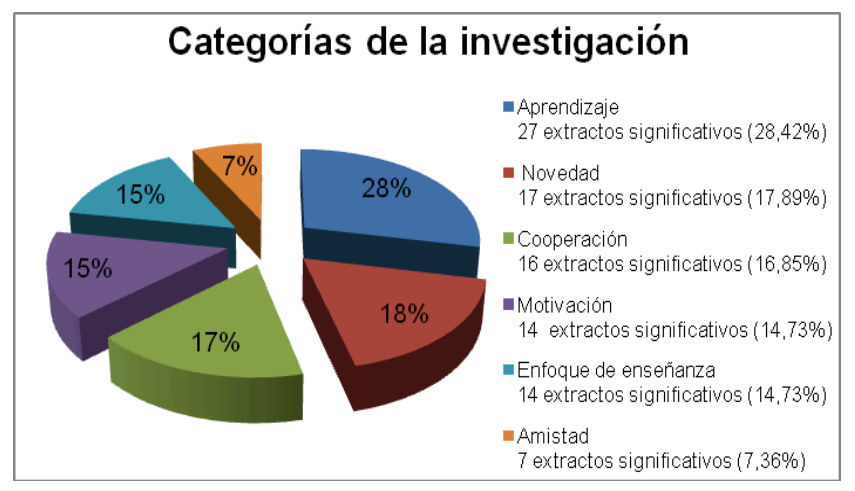

Figura 2. Proporción de datos cualitativos cualitativos de las categorías del estudio

Aprendizaje. El aprendizaje ha sido el aspecto más señalado por todos los participantes tras la realización de la intervención experimental, apareciendo en las entrevistas al alumnado y pregunta abierta y con una frecuencia de extractos significativos de $27(28,42 \%)$. En esta categoría se incluyen todos los comentarios de los participantes que tienen que ver con su experiencia en el aprendizaje de diferentes aspectos tras realizar la intervención basada en MALL. Todos los participantes señalaron que la intervención basada en MALL los ayudó en un aspecto u otro de su aprendizaje. Estos aspectos van desde el aprendizaje en general hasta elementos más concretos. Uno de los elementos más recurrentes apuntados por los participantes fue la adquisición de vocabulario: "El aprendizaje en general. Vocabulario más que otra cosa" (P., entrevistas al alumnado). Algunos participantes comentaron que la intervención de MALL los ayudó en el aprendizaje de gramática: "Aprendes bastante vocabulario, también expresiones como phrasal verbs y cosas así que está muy bien y gramática también" (L., entrevistas al alumnado). Siguiendo la misma línea de aprendizaje lingüístico, otra de las habilidades en la que los participantes manifestaron haber aprendido es la comprensión oral: "A la comprensión oral la parte de podcasts me ayudó bastante porque me tuve que fijar en lo que decían y tal. Entonces en la comprensión oral sí que me ha ayudado" (P., entrevistas al alumnado). Del mismo modo, también parece que la intervención de MALL ayudó en el desarrollo de la expresión oral: "Yo creo que [ayudó] a la expresión oral un poco porque al hacer los videos estos te quitas como el miedo a hablar porque lo pueden ver todos los de la clase" (C., entrevistas al alumnado). Un aspecto fundamental en el aprendizaje de una lengua es ser capaz de entender y manejar la información de diferentes formas. En este sentido la intervención ha favorecido la mejora de esta habilidad: "Aprendí a hacer un telediario y también cómo escuchar y luego resumir y algunas palabras nuevas también" (A., entrevistas al alumnado). Un aspecto valorado positivamente por los participantes fue el "aprender haciendo", como alternativa a que el profesorado explique y el alumnado haga. Los participantes lo consideraron un factor que ayudó a facilitar el aprendizaje: "Porque además de que es más ameno pues no se hace lo típico de escribir en la libreta y luego estudiar. Lo vas aprendiendo mientras lo vas usando y entonces es mucho más fácil" (P., entrevistas al alumnado).

Novedad. La novedad ha sido uno de los aspectos más señalados por el alumnado tras la realización de la intervención experimental, apareciendo en las entrevistas al alumnado y pregunta abierta y con una frecuencia de extractos significativos de 17 $(17,89 \%)$. Esta categoría tiene que ver con el aspecto novedoso resultante de la utilización de dispositivos móviles en la enseñanza de inglés. En ese sentido, el alumnado destaca la originalidad de la experiencia: "Es innovador, es original, es intuitivo, útil y te involucra mucho" (D., pregunta abierta). El hecho de poder trabajar con dispositivos móviles fue muy novedoso para los participantes, dado que es un instrumento muy utilizado por ellos en sus momentos de ocio, y poder usarlo en el aula fue muy provechoso: "Es una gran experiencia ya que no estamos acostumbrados a trabajar con teléfonos móviles en clase y el descubrimiento de esto fue un acierto" (A., pregunta abierta). Del mismo modo, esta nueva forma de trabajar fue apreciada por los participantes como algo adaptado a los tiempos que corren y, por tanto, se valoró como una experiencia útil para repetir: "Me parece un sistema innovador y que corresponde con la tecnología actual por lo que desde luego lo recomendaría en un futuro" (A., pregunta abierta). Los participantes señalaron, además, que les gusta cambiar y hacer actividades diferentes, siempre ayuden a aprender mejor: "Es algo diferente a lo que solemos hacer y a mí lo diferente me gusta, sobre todo lo que funciona bien. $\mathrm{Y}$ esta me pareció que funciona muy bien" (T., 
entrevistas al alumnado). La intervención basada en MALL ha favorecido que los participantes se sientan más involucrados a la hora de participar gracias a la originalidad que ha supuesto para ellos la inclusión de los dispositivos móviles en la clase de inglés: "También es original aprender con cosas que usualmente usamos para jugar $\mathrm{o}$ hablar, $\mathrm{y}$ personalmente, me siento genial sabiendo que nuestros profesores están intentando hacer del aprendizaje una experiencia más satisfactoria; me hace pensar que realmente importamos" (P., pregunta abierta).

Cooperación. La cooperación ha sido uno de los aspectos más señalados por todos los participantes tras la realización de la intervención experimental, apareciendo en el diario del profesor, entrevistas al alumnado y pregunta abierta y con una frecuencia de extractos significativos de $16 \quad(16,85 \%)$. Esta categoría se refiere a las percepciones que los participantes han tenido con relación a la colaboración que la intervención basada en MALL supuso para ellos. En diferentes ocasiones, tanto la profesora participante como el alumnado se refirieron a ella por la relevancia que tuvo en la realización de las actividades. Los participantes manifestaron tener la sensación de que MALL ha facilitado la cooperación y el trabajo en grupo: "Hay un aspecto positivo sobre esto: trabajamos siempre con otros compañeros y creo que eso hace la asignatura más divertida (M., pregunta abierta). La idea de grupo estuvo muy latente, ya que en el momento en el que surgía un problema en el mismo, los miembros se volcaban a resolverlo para poder continuar trabajando, dejando más de lado el individualismo y colaborando unos con otros: "La cooperación e interacción han estado de manifiesto, debido probablemente a que se hace necesaria por el trabajo en grupo. Cada vez que algún miembro del grupo se encuentra con un problema, todos se concentran en resolverlo para poder continuar con la actividad" (diario del profesor, sesión 4), La idea de "ayuda" también aparece repetidamente en las percepciones de los participantes. Se tiene una idea generalizada de sentimiento de haber colaborado y haberse ayudado unos a otros: "Los alumnos interactúan entre ellos y se ayudan cuando un miembro de la pareja no entiende una parte de la entrevista el otro miembro ayuda y viceversa" (diario del profesor, sesión 6), "El contenido, es decir, la teoría parece que pasa a segundo plano por la forma de dar la teoría, lo que pienso que es algo muy positivo porque aprendemos mucho con actividades divertidas donde nos hemos tenido que ayudar los unos a los otros para poder llevar a cabo los trabajos" (R., pregunta abierta).
Motivación. La motivación ha sido uno de los aspectos más señalados por todos los participantes tras la realización de la intervención experimental, apareciendo en el diario del profesor, entrevistas al alumnado y pregunta abierta y con una frecuencia de extractos significativos de 14 (14,73\%). La categoría motivación engloba los comentarios de los participantes en cuanto a los cambios o sensaciones relacionadas con los niveles de motivación de los participantes. A lo largo de las diferentes sesiones, la profesora ha recogido las percepciones en relación a la motivación: "El ambiente motivador es significativo porque en la sesión de hoy se ha generalizado a algunos alumnos que normalmente no lo están tanto. Suele haber un grupo que sí está bastante motivado, pero en la sesión de hoy se ha visto que se ha generalizado aún más" (diario del profesor, sesión 7). Los participantes manifestaron el aumento en su motivación e interés por la clase de inglés, y atribuyen a este incremento de la motivación y el interés a diferentes factores como el hecho de utilizar el móvil, trabajar en grupo o pasar más tiempo con los amigos: "Yo creo que toda la clase ha retomado el interés en aprender inglés y el trabajar en grupo y estar con los amigos que yo creo que apetece" (R., entrevistas al alumnado). El factor de novedad previamente mencionado en este apartado tuvo un importante impacto en la motivación de los participantes, y estos lo manifestaron en varias ocasiones: "Bueno, yo creo que al ser una forma diferente pues la gente se motiva más porque están acostumbrados a teoría y luego ejercicios pues es una nueva forma y más entretenida" (M., entrevistas al alumnado). La motivación ha contribuido además a fomentar el aprendizaje, ya que al sentirse más motivados los participantes tienen también más ganas de aprender. Los participantes reflejaron que las clases fueron más amenas, lo cual llevó a que se divirtiesen a la vez que aprendían: "Además de conocer nuevos conceptos, lo pasé muy bien y estaría genial que en todas las asignaturas tuviéramos una unidad igual. Nos motiva, nos entretiene y nos culturiza de manera diferente" (D., pregunta abierta).

Enfoque de enseñanza. La metodología también ha sido uno de los aspectos más señalados por todos los participantes tras la realización de la intervención experimental, apareciendo en las entrevistas al alumnado, pregunta abierta y diario del profesor y con una frecuencia de extractos significativos de 14 $(14,73 \%)$. En este apartado el enfoque de enseñanza se refiere a la metodología utilizada en la intervención basada en MALL e incluye los comentarios de los participantes en relación con el método de aprendizaje utilizado. La metodología 
utilizada fue señalada por los participantes en diferentes ocasiones como una forma más entretenida y divertida de aprender. Se sintieron en general más comprometidos y entretenidos con la manera de aprender utilizando MALL: "Es mucho menos aburrido y te involucra mucho más, es más divertido y más dinámico" (J., entrevistas al alumnado). En relación con los contenidos, los participantes manifestaron que el método de aprendizaje utilizado facilitó la adquisición de contenidos a través de una metodología diferente: "Los contenidos se quedan mejor que habitualmente por el tema de que es mucho más didáctico e involucrativo" (M., entrevistas al alumnado). El enfoque de enseñanza utilizado en la intervención puesta en práctica en este estudio permitió a los participantes descubrir nuevas formas de uso de sus dispositivos móviles en el ámbito del aprendizaje de lenguas extranjeras: "He descubierto cómo utilizar mi teléfono móvil de una forma diferente. Antes de esto, solo lo utilizaba para navegar por Internet y para jugar a juegos, pero ahora sé que lo puedo utilizar para aprender inglés" (M., pregunta abierta). Para todos los participantes el descubrimiento del enfoque MALL fue una experiencia muy positiva que les gustaría repetir: "Creo que es la mejor forma de aprender; el enfoque tradicional se hace repetitivo con el paso del tiempo y te aburre. El m-learning es la mejor forma de aprender" (R., pregunta abierta). Por otro lado, los participantes señalaron el mal funcionamiento de Internet como un inconveniente a la hora de trabajar: "Que hubiese funcionado mejor Internet para poder aprovechar más el tiempo" (D., entrevistas al alumnado). La profesora también percibió el mal funcionamiento de Internet como algo negativo: "Se nota frustración cuando Internet no funciona bien" (diario del profesor, sesión 3).

Amistad. La amistad ha sido otro de los aspectos señalados por todos los participantes, apareciendo en el diario del profesor, entrevistas al alumnado y pregunta abierta y con una frecuencia de extractos significativos de 7 (7,36\%). Esta categoría está relacionada con las relaciones personales que surgieron o se afianzaron durante la intervención basada en MALL y en ella se muestran los comentarios de los participantes relacionados con este aspecto. La intervención basada en el MALL, gracias también al diseño colaborativo de las actividades, parece haber favorecido la relación entre los compañeros de clase. La relación entre las personas que ya tenían trato parece haber mejorado y también se establecieron nuevos vínculos entre personas que anteriormente no eran muy cercanas: "El ambiente de trabajo y la relación entre los compañeros ha sido mejor" (P., entrevistas al alumnado). La formación de los grupos y la necesidad de trabajar de forma colaborativa parece haber facilitado la creación de lazos entre personas: "Como casi todas las pruebas son con compañeros y tienen que ser todos diferentes haces más amistades y más relación" (M., entrevistas al alumnado). Uno de los inconvenientes de las clases tradicionales, según apuntaron los participantes, es la falta de tiempo que existe normalmente para establecer o consolidar relaciones dentro del horario lectivo. No obstante, gracias al MALL y al diseño pedagógico de las actividades se puede apreciar un cambio a este respecto: "Al trabajar en grupo, lo creas o no la confianza mejora y luego eso se nota en más trabajos y es una buena forma de mejorar las relaciones" (M., entrevista al alumnado). La formación de diferentes grupos favoreció la necesidad de relacionarse con personas que no siempre son del mismo círculo. Se abrieron oportunidades de conocer o profundizar en relaciones con personas con las que anteriormente no habían tenido ocasión de hablar: "Me sorprende la buena relación entre algunos miembros de la clase que anteriormente no habían establecido ningún contacto en la clase de inglés" (diario del profesor, sesión 13). "Además trabajando en grupos puedes afianzar relación o crear relaciones con gente de clase con la que no tenías tanta relación o apenas no tenías relación" (M., entrevistas al alumnado).

\section{DISCUSIÓN Y CONCLUSIONES}

El objetivo de este estudio fue conocer las percepciones de alumnado y profesorado sobre una intervención basada en MALL en la asignatura de ILE en la etapa de educación secundaria.

Los resultados reflejan que el aprendizaje fue la categoría más reiterada por los participantes en los diferentes instrumentos de recogida de datos. Según las percepciones de los participantes, la intervención basada en MALL les ayudó en la adquisición de vocabulario, gramática, comprensión oral y escrita expresión oral, capacidad organizativa y de trabajo en equipo, y pensamiento autónomo. En este sentido, los resultados concuerdan con estudios anteriores como el Hsu et al. (2013), que señala mejoras en la comprensión lectora tras una aplicación de una intervención de MALL. Según los participantes, la experiencia les ha ayudado especialmente en el aprendizaje de vocabulario. Esto coincide con estudios realizados por Saran, Cagiltay, y Seferoglu (2008), Lu (2008) y Kukulska-Hulme y Shield (2008), en los que se demostró que los participantes se mostraban favorables a la utilización de dispositivos móviles para el aprendizaje de 
vocabulario y en los que los resultados fueron positivos. Esta categoría tiene una gran importancia, ya que desvela que el uso de MALL puede contribuir positivamente al aprendizaje de diferentes aspectos básicos en la adquisición de una lengua.

El aspecto de la novedad fue uno de los aspectos más relevantes percibido por los participantes. Estos valoraron muy positivamente el hecho de utilizar una nueva forma de aprender utilizando herramientas tan familiares para ellos como son los dispositivos móviles. Asimismo, manifestaron que el hecho de utilizar estos dispositivos les parecía un método muy actual. Esto enlaza con el estudio llevado a cabo por Jones y Issroff (2007), en el que también se refieren al uso de los dispositivos móviles como entretenimiento, lo cual puede por sí mismo crear emoción y excitación solo por el hecho de utilizarlos. Estos datos revelan que la novedad es algo a tener en cuenta a la hora de diseñar metodologías interesantes para el alumnado.

La cooperación fue otra de las categorías relevantes del estudio. Todos los participantes afirmaron haber percibido un aumento en su motivación y en su interés por la asignatura tras la intervención basada en MALL. Asimismo, la cooperación para la realización de las actividades fue altamente valorada por los participantes, quienes consideraron las actividades utilizando MALL como entretenidas y divertidas. Jones y Issroff (2007) y Monguillot et al. (2014) subrayan que los dispositivos móviles permiten a los usuarios comunicarse, facilitando el aprendizaje colaborativo y cooperativo. Con esta información se puede afirmar que la cooperación va muy ligada a la motivación, ambas esenciales para que el alumnado perciba la experiencia educativa como algo útil y relevante.

La motivación apareció en los diferentes instrumentos de recogida de datos. Todos los participantes afirman haber percibido un aumento en su motivación tras la intervención basada en MALL. Estos resultados coinciden con los de Alamer (2015) y Hsu et al. (2013), quienes afirman que si el aprendizaje móvil de lenguas es utilizado correctamente puede aumentar la motivación del alumnado de lenguas extranjeras. Su estudio se basó en el uso informal de la tecnología móvil. Los resultados del estudio reflejaron que los participantes mostraron actitudes positivas hacia el uso de dispositivos móviles.

El enfoque de enseñanza fue otra de las categorías que emergió en el análisis de datos, apareciendo en las entrevistas al alumnado, en la pregunta abierta y en el diario del profesor. El hecho de utilizar un enfoque de enseñanza novedoso que, además utiliza la tecnología móvil, resultó más motivador para los participantes. Los participantes apuntaron que el hecho de poder utilizar de manera didáctica elementos que normalmente utilizan en su vida cotidiana para comunicarse o divertirse fue un acierto. Esto coincide con las con los resultados del estudio de Keengwe y Bhargava (2014) en los que señalan que las tecnologías móviles tienen mucho potencial para transformar la educación cuando se diseñan y se ponen en práctica de manera que sean relevantes para el contexto cultural y social del alumnado. De estos datos se extrae la necesidad de incluir las tecnologías móviles para crear entornos de aprendizaje relevantes para el alumnado.

La categoría amistad, aunque de menor relevancia que las anteriores, apareció en los distintos instrumentos de recogida de datos. Los participantes señalaron haber tenido oportunidad de establecer nuevas relaciones con personas con las que no tenían mucha relación, y afianzar la amistad con personas con las que ya la tenían. En ese sentido, en línea con otros estudios (Crook, 2000), trabajar con otros estudiantes de forma colaborativa ayudar a mejorar la motivación, fomentar las relaciones personales y saber expresarse y aceptar las ideas expresadas por otros. Estos datos nos dan una visión de la importancia de incluir el trabajo colaborativo para mejorar la satisfacción en el aprendizaje del alumnado y mejorar las relaciones sociales, y el aprendizaje móvil, en ese sentido, facilita el diseño de actividades colaborativas.

Las principales limitaciones del estudio se encuentran en el escaso número de participantes y grupos participantes Futuras investigaciones deberán llevarse con más participantes, en más centros y con más grupos. Otra de las limitaciones es que la intervención solo se llevó a cabo en un nivel educativo. Futuros estudios deberán analizar otros cursos de la educación secundaria para comprobar si se producen resultados similares. Finalmente, cabe mencionar que en ocasiones la conexión a Internet ocasionó problemas, lo cual dificultó el correcto desarrollo de algunas sesiones.

A modo de conclusión, el presente estudio ha reflejado las positivas percepciones de alumnado y profesorado tras la aplicación de un programa de MALL en la asignatura de ILE en la etapa de educación secundaria. Los resultados reflejaron cómo la intervención ayudó al aprendizaje del inglés a diferentes niveles (adquisición de vocabulario, comprensión, gramática, etc.), mejoró la cooperación y colaboración entre el alumnado, fortaleció sus vínculos de amistad y además fue considerado como una experiencia diferente, novedosa, motivadora y que rompe con la rutina 
tradicional de esta asignatura. Estos resultados muestran los beneficios y potencialidades que tiene el aprendizaje móvil en el aula de ILE.

\section{REFERENCIAS}

Alamer, A. (2015). The Role of EFL Learners' Motivation in Mobile Language Learning. Comunicación presentada en el First International Conference on Theory and Practice. Adelaida: Australia.

Amador, E., y Pantoja, A. (2017). Eficacia de un programa de intervención basado en el uso de las TIC en tutorías. Revista de Investigación Educativa, 35(1), 215-333. DOI: http://dx.doi.org/10.6018/rie.35.1.248831

Arashnia, M., y Shahrokhi, M. (2016). Mobile Assisted Language Learning: English Pronunciation among Iranian Pre-intermediate EFL Learners. Journal of Applied Linguistics and Language Research, 3(4), 149162.

Attewell, J. (2005). Mobile technologies and learning: A technology update and m-learning project summary. London, UK: Learning Skills Development Agency.

Bisquerra, R. (coord.) (2012). Metodología de la investigación educativa. Madrid: La Muralla.

Cantillo, C., Roura, M., y Sánchez, A. (2012). Tendencias actuales en el uso de dispositivos móviles en educación. La Educación Digital Magazine, 147, 1-21.

Coll, C. (2013). El currículo escolar en el marco de la nueva ecología del aprendizaje. Aula, 219, 31-36.

Conole, G. (2016). MOOC as disruptive technologies: strategies for enhancing the learner experience and quality of MOOCs. Revista de Educación a Distancia, 50(2), 1-18. DOI: http://dx.doi.org/10.6018/red/50/2

Crook, C. (2000). Motivation and the ecology of collaborative learning. En R. Joiner (Ed.), Rethinking collaboration. Londres: Routledge.

Denzin, N. K., y Lincoln, Y. S. (1994). Handbook of qualitative research. Thousand Oaks, CA: Sage. DOI: http://dx.doi.org/10.4135/9781848607927

Fombona, J., y Pascual, M. A. (2013). Beneficios del m-learning en la Educación Superior. Educatio Siglo XXI, 31(2), 211-234.

García-Peñalvo, F. J., y Seoane, A. M. (2015). Una revisión actualizada del concepto de eLearning. Décimo Aniversario. Teoría de la Educación. Educación y Cultura en la Sociedad de la Información, 16(1), 119144.

DOI: http://dx.doi.org/10.14201/eks2015161119144

Hsu, C., Hwang, G., y Chang., C. (2013). A personalized recommendation-based mobile learning approach to improving the reading performance of EFL students. Computers y Education, 63, 327-336. DOI: http://dx.doi.org/10.1016/j.compedu.2012.12.004

Illanas, A., y Llorens, F. (2011). Los retos Web 2.0 de cara al EEES. En C. Suarez- Guerrero, y F. J. Garcia-Penalvo (Eds.), Universidad y Desarrollo Social de la Web (pp. 13-34): Washington: Editandum.

Jones, M. e Issroff, K. (2007). Motivation and mobile devices: exploring the role of appropriation and coping strategies. Research in Learning Technology, 15(3), 247-258.

Keengwe, J., y Bhargava, M. (2014). Mobile learning and integration of mobile technologies in education. Education and Information Technologies, 19(4), 737746. DOI: http://dx.doi.org/10.1007/s10639-013-9250-3
Klopfer, E., y Squire, K. (2008). Environmental Detectives: the development of an augmented reality platform for environmental simulations. Educational Technology Research and Development, 56(2), 203-228. DOI: http://dx.doi.org/10.1007/s11423-007-9037-6

Kramsch, C., A'Ness, F., y Lam, E. (2000). Authenticity and authorship in the computer mediated acquisition of L2 literacy. Language Learning y Technology, 4(2), 78104.

Kukulska-Hulme, A., y Shield, L. (2008). An overview of mobile assisted language learning: From content delivery to supported collaboration and interaction. ReCALL, 20(3), 271-289. DOI: http://dx.doi.org/10.1017/S0958344008000335

Lan, Y., Sung, Y., y Chang, K. (2007). A mobile-devicesupported peer-assisted learning system for collaborative early EFL reading, Language Learning and Technology, 11(3), 130-151.

Lee, H., y Lee, J. H. (2013). Implementing glossing in mobileassisted language learning environments: Directions and outlook. Language Learning \& Technology, 17(3), 6-22

Libarkin, J. C., y Kurdziel, J. P. (2002). Research methodologies in science education: the qualitative-quantitative debate. Journal of Geoscience Education, 50(1), 78- 86.

Lincoln, Y. S., y Guba, E. G. (1985). Naturalistic inquiry. Newbury Park, CA: Sage. DOI: http://dx.doi.org/10.1177/1468794107085301

Liu, J., Yu, S., y Ran, M. (2008). Research on the communicative mobile English learning model. En Fifth IEEE international conference on wireless, mobile, and ubiquitous technology in education (pp. 60-64). China: Wuhan.

Lu M. (2008). Effectiveness of vocabulary learning via mobile phone. National Keelung Vocational High School. Journal of Computer Assisted Learning, 24(6), 515-525. DOI: $\quad$ http://dx.doi.org/10.1111/j.13652729.2008.00289.x

Monguillot, M., González, C., Guitert, M., y Zurita, C. (2014). Mobile learning: a collaborative experience using QR codes. Revista de Universidad y Sociedad del Conocimiento, 11(1), DOI: http://dx.doi.org/10.7238/rusc.v11i1.1899

Mulatu, M. (2015). Language Learning through Mobile Technologies: An Opportunity for Language Learners and Teachers. Journal of Education and Practice, 6(31), 50-53.

Pastor, A., y Zubillaga, A. (2012). La utilización de las TICs en la actividad académica de los estudiantes universitarios con discapacidad. Revista Complutense de Educación, 23(1), 23-49

Saldaña, J. (2009). The Coding Manual for Qualitative Researchers. Thousand Oaks, CA: Sage.

Saran, M., Cagiltay, K., y Seferoglu, G. (2008). Use of mobile phones in language learning: Developing effective instructional materials. Actas del Fifth IEEE International Conference on Wireless, Mobile, and Ubiquitous Technology in Education. Washington: USA. DOI: http://dx.doi.org/10.1109/WMUTE.2008.49

Shih, Y., y Mills, D. (2007). Setting the New Standard with Mobile Computing in Online Learning. International Review of Research in Open and Distance Learning, $8(2), \quad 1-16 . \quad$ DOI: http://dx.doi.org/10.19173/irrodl.v8i2.361

Villalonga, C., y Marta-Lazo, C. (2015). Modelo de integración educomunicativa de "apps" móviles para la enseñanza y el aprendizaje. Pixel-Bit, 46, 137-153. DOI: http://dx.doi.org/10.12795/pixelbit.2015.i46.09 